\title{
Fixed points of multivalued contraction mappings in modular metric spaces
}

\author{
Afrah AN Abdou ${ }^{1 *}$ and Mohamed A Khamsi ${ }^{2,3}$
}

"Correspondence:

aabdou@kau.edu.sa

${ }^{1}$ Department of Mathematics, King Abdulaziz University, P.O. Box 80203,

Jeddah, 21589, Saudi Arabia

Full list of author information is

available at the end of the article

\begin{abstract}
The purpose of this paper is to study the existence of fixed points for contractive-type multivalued maps in the setting of modular metric spaces. The notion of a modular metric on an arbitrary set and the corresponding modular spaces, generalizing classical modulars over linear spaces like Orlicz spaces, were recently introduced. In this paper we investigate the existence of fixed points of multivalued modular contractive mappings in modular metric spaces. Consequently, our results either generalize or improve fixed point results of Nadler (Pac. J. Math. 30:475-488, 1969) and Edelstein (Proc. Am. Math. Soc. 12:7-10, 1961).

MSC: Primary 47H09; secondary 46B20; 47H10; 47E10
\end{abstract}

Keywords: $\Delta_{2}$-condition; fixed point; modular metric spaces; multivalued contraction mapping

\section{Introduction}

The aim of this paper is to give an outline of a fixed point theory for multivalued Lipschitzian mappings defined on some subsets of modular metric spaces. Modular metric spaces were introduced in $[1,2]$. The way we approached the concept of modular metric spaces is different. Indeed we look at these spaces as the nonlinear version of the classical modular spaces as introduced by Nakano [3] on vector spaces and modular function spaces introduced by Musielak [4] and Orlicz [5]. In [6] the authors have defined and investigated the fixed point property in the framework of modular metric space and introduced the analog of the Banach contraction principle theorem in modular metric space.

As is well known, a fixed point theorem for multivalued contraction mappings was established by Nadler [7]. In 1961 Edelstein [8] has generalized the Banach contraction principle to mappings satisfying a less restrictive Lipschitz inequality such as local contraction. This result has been generalized to a multivalued version by Nadler [7]. On the other hand Mizoguchi and Takahashi [9] have improved Reich's result [10] and proved the existence of fixed points for multivalued maps in the case when values of mappings are closed bounded instead of compact.

In this paper we define the Hausdorff modular metric and obtain a multivalued version of the result [6, Theorem 3.1] in modular metric spaces. We also extend the results of Nadler [7], Mizoguchi and Takahashi [9] to modular metric spaces. The linear version of some of our results may be found in the work of Kutbi and Latif [11].

For more on metric fixed point theory, the reader may consult the book [12].

02014 Abdou and Khamsi; licensee Springer. This is an Open Access article distributed under the terms of the Creative Commons Attribution License (http://creativecommons.org/licenses/by/2.0), which permits unrestricted use, distribution, and reproduction in any medium, provided the original work is properly cited. 


\section{Basic definitions and properties}

Let $X$ be a nonempty set. Throughout this paper for a function $\omega:(0, \infty) \times X \times X \rightarrow$ $(0, \infty)$, we will write

$$
\omega_{\lambda}(x, y)=\omega(\lambda, x, y)
$$

for all $\lambda>0$ and $x, y \in X$.

Definition 2.1 $[1,2]$ A function $\omega:(0, \infty) \times X \times X \rightarrow[0, \infty]$ is said to be a modular metric on $X$ if it satisfies the following axioms:

(i) $x=y$ if and only if $\omega_{\lambda}(x, y)=0$, for all $\lambda>0$;

(ii) $\omega_{\lambda}(x, y)=\omega_{\lambda}(y, x)$, for all $\lambda>0$, and $x, y \in X$;

(iii) $\omega_{\lambda+\mu}(x, y) \leq \omega_{\lambda}(x, z)+\omega_{\mu}(z, y)$, for all $\lambda, \mu>0$ and $x, y, z \in X$.

If instead of (i), we have only the condition

(i') $\omega_{\lambda}(x, x)=0, \quad$ for all $\lambda>0, x \in X$,

then $\omega$ is said to be a pseudomodular (metric) on $X$. A modular metric $\omega$ on $X$ is said to be regular if the following weaker version of (i) is satisfied:

$x=y \quad$ if and only if $\omega_{\lambda}(x, y)=0, \quad$ for some $\lambda>0$.

Finally, $\omega$ is said to be convex if for $\lambda, \mu>0$ and $x, y, z \in X$, it satisfies the inequality

$$
\omega_{\lambda+\mu}(x, y) \leq \frac{\lambda}{\lambda+\mu} \omega_{\lambda}(x, z)+\frac{\mu}{\lambda+\mu} \omega_{\mu}(z, y) .
$$

Note that for a metric pseudomodular $\omega$ on a set $X$, and any $x, y \in X$, the function $\lambda \rightarrow$ $\omega_{\lambda}(x, y)$ is nonincreasing on $(0, \infty)$. Indeed, if $0<\mu<\lambda$, then

$$
\omega_{\lambda}(x, y) \leq \omega_{\lambda-\mu}(x, x)+\omega_{\mu}(x, y)=\omega_{\mu}(x, y) .
$$

Definition $2.2[1,2]$ Let $\omega$ be a pseudomodular on $X$. Fix $x_{0} \in X$. The two sets

$$
X_{\omega}=X_{\omega}\left(x_{0}\right)=\left\{x \in X: \omega_{\lambda}\left(x, x_{0}\right) \rightarrow 0 \text { as } \lambda \rightarrow \infty\right\}
$$

and

$$
X_{\omega}^{*}=X_{\omega}^{*}\left(x_{0}\right)=\left\{x \in X: \exists \lambda=\lambda(x)>0 \text { such that } \omega_{\lambda}\left(x, x_{0}\right)<\infty\right\}
$$

are said to be modular spaces (around $\left.x_{0}\right)$.

We obviously have $X_{\omega} \subset X_{\omega}^{*}$. In general this inclusion may be proper. It follows from $[1,2]$ that if $\omega$ is a modular on $X$, then the modular space $X_{\omega}$ can be equipped with a (nontrivial) metric, generated by $\omega$ and given by

$$
d_{\omega}(x, y)=\inf \left\{\lambda>0: \omega_{\lambda}(x, y) \leq \lambda\right\}
$$


for any $x, y \in X_{\omega}$. If $\omega$ is a convex modular on $X$, according to $[1,2]$ the two modular spaces coincide, i.e. $X_{\omega}^{*}=X_{\omega}$, and this common set can be endowed with the metric $d_{\omega}^{*}$ given by

$$
d_{\omega}^{*}(x, y)=\inf \left\{\lambda>0: \omega_{\lambda}(x, y) \leq 1\right\}
$$

for any $x, y \in X_{\omega}$. These distances will be called Luxemburg distances.

First attempts to generalize the classical function spaces of the Lebesgue type $L^{p}$ were made in the early 1930s by Orlicz and Birnbaum in connection with orthogonal expansions. Their approach consisted in considering spaces of functions with some growth properties different from the power type growth control provided by the $L^{p}$-norms. Namely, they considered the function spaces defined as follows:

$$
L^{\varphi}=\left\{f: \mathbb{R} \rightarrow \mathbb{R} ; \exists \lambda>0: \rho(\lambda f)=\int_{\mathbb{R}} \varphi(\lambda|f(x)|) d x<\infty\right\}
$$

where $\varphi:[0, \infty] \rightarrow[0, \infty]$ was assumed to be a convex function increasing to infinity, i.e. the function which to some extent behaves similarly to power functions $\varphi(t)=t^{p}$. Modular function spaces $L^{\varphi}$ furnishes a wonderful example of a modular metric space. Indeed define the function $\omega$ by

$$
\omega_{\lambda}(f, g)=\rho\left(\frac{f-g}{\lambda}\right)=\int_{\mathbb{R}} \varphi\left(\frac{|f(x)-g(x)|}{\lambda}\right) d x,
$$

for all $\lambda>0$, and $f, g \in L^{\varphi}$. Then $\omega$ is a modular metric on $L^{\varphi}$. Moreover, the distance $d_{\omega}^{*}$ is exactly the distance generated by the Luxemburg norm on $L^{\varphi}$.

For more examples on modular function spaces, the reader may consult the book of Kozlowski [13] and for modular metric spaces [1,2].

Definition 2.3 Let $X_{\omega}$ be a modular metric space.

(1) The sequence $\left\{x_{n}\right\}_{n \in \mathbb{N}}$ in $X_{\omega}$ is said to be $\omega$-convergent to $x \in X_{\omega}$ if and only if $\omega_{1}\left(x_{n}, x\right) \rightarrow 0$, as $n \rightarrow \infty$. $x$ will be called the $\omega$-limit of $\left\{x_{n}\right\}$.

(2) The sequence $\left\{x_{n}\right\}_{n \in N}$ in $X_{\omega}$ is said to be $\omega$-Cauchy if $\omega_{1}\left(x_{m}, x_{n}\right) \rightarrow 0$, as $m, n \rightarrow \infty$.

(3) A subset $M$ of $X_{\omega}$ is said to be $\omega$-closed if the $\omega$-limit of a $\omega$-convergent sequence of $M$ always belongs to $M$.

(4) A subset $M$ of $X_{\omega}$ is said to be $\omega$-complete if any $\omega$-Cauchy sequence in $M$ is a $\omega$-convergent sequence and its $\omega$-limit is in $M$.

(5) A subset $M$ of $X_{\omega}$ is said to be $\omega$-bounded if we have

$$
\delta_{\omega}(M)=\sup \left\{\omega_{1}(x, y) ; x, y \in M\right\}<\infty .
$$

(6) A subset $M$ of $X_{\omega}$ is said to be $\omega$-compact if for any $\left\{x_{n}\right\}$ in $M$ there exists a subset sequence $\left\{x_{n_{k}}\right\}$ and $x \in M$ such that $\omega_{1}\left(x_{n_{k}}, x\right) \rightarrow 0$.

(7) $\omega$ is said to satisfy the Fatou property if and only if for any sequence $\left\{x_{n}\right\}_{n \in \mathbb{N}}$ in $X_{\omega}$ $\omega$-convergent to $x$, we have

$$
\omega_{1}(x, y) \leq \liminf _{n \rightarrow \infty} \omega_{1}\left(x_{n}, y\right)
$$

for any $y \in X_{\omega}$. 
In general if $\lim _{n \rightarrow \infty} \omega_{\lambda}\left(x_{n}, x\right)=0$, for some $\lambda>0$, then we may not have $\lim _{n \rightarrow \infty} \omega_{\lambda}\left(x_{n}\right.$, $x)=0$, for all $\lambda>0$. Therefore, as is done in modular function spaces, we will say that $\omega$ satisfies the $\Delta_{2}$-condition if this is the case, i.e. $\lim _{n \rightarrow \infty} \omega_{\lambda}\left(x_{n}, x\right)=0$, for some $\lambda>0$ implies $\lim _{n \rightarrow \infty} \omega_{\lambda}\left(x_{n}, x\right)=0$, for all $\lambda>0$. In [1] and [2], one will find a discussion as regards the connection between $\omega$-convergence and metric convergence with respect to the Luxemburg distances. In particular, we have

$$
\lim _{n \rightarrow \infty} d_{\omega}\left(x_{n}, x\right)=0 \quad \text { if and only if } \quad \lim _{n \rightarrow \infty} \omega_{\lambda}\left(x_{n}, x\right)=0, \quad \text { for all } \lambda>0,
$$

for any $\left\{x_{n}\right\} \in X_{\omega}$ and $x \in X_{\omega}$. In particular we see that $\omega$-convergence and $d_{\omega}$-convergence are equivalent if and only if the modular $\omega$ satisfies the $\Delta_{2}$-condition. Moreover, if the modular $\omega$ is convex, then we know that $d_{\omega}^{*}$ and $d_{\omega}$ are equivalent, which implies

$$
\lim _{n \rightarrow \infty} d_{\omega}^{*}\left(x_{n}, x\right)=0 \quad \text { if and only if } \quad \lim _{n \rightarrow \infty} \omega_{\lambda}\left(x_{n}, x\right)=0, \quad \text { for all } \lambda>0,
$$

for any $\left\{x_{n}\right\} \in X_{\omega}$ and $x \in X_{\omega}[1,2]$.

Definition 2.4 Let $(X, \omega)$ be a modular metric space. We will say that $\omega$ satisfies the $\Delta_{2}$ type condition if, for any $\alpha>0$, there exists $C>0$ such that

$$
\omega_{\lambda / \alpha}(x, y) \leq C \omega_{\lambda}(x, y)
$$

for any $\lambda>0, x, y \in X_{\omega}$, with $x \neq y$.

Note that if $\omega$ satisfies $\Delta_{2}$-type condition, then $\omega$ satisfies the $\Delta_{2}$-condition. The above definition will allow us to introduce the growth function in the modular metric spaces as was done in the linear case.

Definition 2.5 Let $(X, \omega)$ be a modular metric space. Define the growth function $\Omega$ by

$$
\Omega(\alpha)=\sup \left\{\frac{\omega_{\lambda / \alpha}(x, y)}{\omega_{\lambda}(x, y)} ; \lambda>0, x, y \in X_{\omega}, x \neq y\right\},
$$

for any $\alpha>0$.

The following properties were proved in the linear case in [14].

Lemma 2.1 Let $(X, \omega)$ be a modular metric space. Assume that $\omega$ is a convex regular modular which satisfies the $\Delta_{2}$-type condition. Then

(1) $\Omega(\alpha)<\infty$, for any $\alpha>0$,

(2) $\Omega$ is a strictly increasing function, and $\Omega(1)=1$,

(3) $\Omega(\alpha \beta) \leq \Omega(\alpha) \Omega(\beta)$, for any $\alpha, \beta \in(0, \infty)$,

(4) $\Omega^{-1}(\alpha) \Omega^{-1}(\beta) \leq \Omega^{-1}(\alpha \beta)$, where $\Omega^{-1}$ is the function inverse of $\Omega$,

(5) for any $x, y \in X_{\omega}, x \neq y$, we have

$$
d_{\omega}^{*}(x, y) \leq \frac{1}{\Omega^{-1}\left(1 / \omega_{1}(x, y)\right)} .
$$


Proof It is clear that the $\Delta_{2}$-type condition implies (1) and from the definition of $\Omega$ we have $\Omega(1)=1$. Next we prove that $\Omega$ is strictly increasing. Let $\alpha<\beta$. Since $\omega$ is convex, we get

$$
\omega_{\alpha+\beta-\alpha}(x, y) \leq \frac{\beta-\alpha}{\alpha+\beta-\alpha} \omega_{\beta-\alpha}(x, x)+\frac{\alpha}{\alpha+\beta-\alpha} \omega_{\alpha}(x, y)
$$

which implies

$$
\omega_{\beta}(x, y) \leq \frac{\alpha}{\beta} \omega_{\alpha}(x, y)
$$

for any $x, y \in X_{\omega}$. From this inequality, we can easily deduce the following relation:

$$
\Omega(\alpha) \leq \frac{\alpha}{\beta} \Omega(\beta),
$$

for any $\alpha<\beta$. The properties (3) and (4) follow the same line as the proofs developed in [14]. As for the property (5), note that

$$
\omega \frac{1}{\Omega^{-1}\left(1 / \omega_{1}(x, y)\right)}(x, y) \leq \Omega\left(\Omega^{-1}\left(1 / \omega_{1}(x, y)\right)\right) \omega_{1}(x, y)=\frac{1}{\omega_{1}(x, y)} \omega_{1}(x, y)=1 .
$$

The inequality in (5) follows from the definition of the distance $d_{\omega}^{*}$.

The following technical lemma will be useful later on in this work.

Lemma 2.2 Let $(X, \omega)$ be a modular metric space. Assume that $\omega$ is a convex regular modular which satisfies the $\Delta_{2}$-type condition. Let $\left\{x_{n}\right\}$ be a sequence in $X_{\omega}$ such that

$$
\omega_{1}\left(x_{n+1}, x_{n}\right) \leq K \alpha^{n}, \quad n=1, \ldots
$$

where $K$ is an arbitrary nonzero constant and $\alpha \in(0,1)$. Then $\left\{x_{n}\right\}$ is Cauchy for both $\omega$ and $d_{\omega}^{*}$.

Proof Under the above assumptions, we have

$$
\frac{1}{K \alpha^{n}} \leq \frac{1}{\omega_{1}\left(x_{n+1}, x_{n}\right)}
$$

where we assumed that $x_{n} \neq x_{n+1}$. Hence

$$
\Omega^{-1}\left(\frac{1}{\omega_{1}\left(x_{n+1}, x_{n}\right)}\right) \geq \Omega^{-1}\left(\frac{1}{K \alpha^{n}}\right) \geq \Omega^{-1}\left(\frac{1}{K}\right)\left(\Omega^{-1}\left(\frac{1}{\alpha}\right)\right)^{n},
$$

for $n \geq 1$, which implies

$$
d_{\omega}^{*}\left(x_{n+1}, x_{n}\right) \leq \frac{1}{\Omega^{-1}\left(1 / \omega_{1}\left(x_{n+1}, x_{n}\right)\right)} \leq \frac{1}{\Omega^{-1}\left(\frac{1}{K}\right)} \frac{1}{\left(\Omega^{-1}\left(\frac{1}{\alpha}\right)\right)^{n}},
$$


for any $n \geq 1$. Note that this inequality is still valid when $x_{n+1}=x_{n}$. Since $\alpha<1$, and $\Omega^{-1}$ is strictly increasing, we get

$$
1=\Omega^{-1}(1)<\Omega^{-1}\left(\frac{1}{\alpha}\right)
$$

which implies $\frac{1}{\Omega^{-1}\left(\frac{1}{\alpha}\right)}<1$. Classical analysis on metric spaces implies that $\sum_{n \geq 1} d_{\omega}^{*}\left(x_{n+1}, x_{n}\right)$ is convergent which implies that $\left\{x_{n}\right\}$ is Cauchy for $d_{\omega}^{*}$. Since $\omega$ satisfies the $\Delta_{2}$-type condition, $\left\{x_{n}\right\}$ is Cauchy for $\omega$.

Note that this lemma is crucial since the main assumption on $\left\{x_{n}\right\}$ will not be enough to imply that $\left\{x_{n}\right\}$ is $\omega$-Cauchy since $\omega$ fails the triangle inequality.

\section{Multivalued mappings in modular metric spaces}

At this point we introduce some notation which will be used throughout the remainder of this work. For a subset $M$ of modular metric space $X_{\omega}$. Set

(i) $C B(M)=\{C: C$ is nonempty $\omega$-closed and $\omega$-bounded subset of $M\}$;

(ii) $K(M)=\{C: C$ is nonempty $\omega$-compact subset of $M\}$;

(iii) the Hausdorff modular metric is defined on $C B(M)$ by

$$
H_{\omega}(A, B)=\max \left\{\sup _{x \in A} \omega_{1}(x, B), \sup _{y \in B} \omega_{1}(y, A)\right\}
$$

where $\omega_{1}(x, B)=\inf _{y \in A} \omega_{1}(x, y)$.

Definition 3.1 Let $(X, \omega)$ be a modular metric space and $M$ be a nonempty subset of $X_{\omega}$. A mapping $T: M \rightarrow C B(M)$ is called a multivalued Lipschitzian mapping if there exists a constant $k \geq 0$ such that

$$
H_{\omega}(T(x), T(y)) \leq k \omega_{1}(x, y), \quad \text { for any } x, y \in M
$$

A point $x \in M$ is called a fixed point of $T$ whenever $x \in T(x)$. The set of fixed points of $T$ will be denoted by $\operatorname{Fix}(T)$.

In [6], we defined Lipschitzian single valued maps. Our definition is more general than the one used by Chistyakov [1, 2]. Indeed, in the case of modular function spaces, it is proved in [15] that

$$
\omega_{\lambda}(T(x), T(y)) \leq \omega_{\lambda}(x, y)
$$

for any $\lambda>0$ if and only if

$$
d_{\omega}(T(x), T(y)) \leq d_{\omega}(x, y)
$$

Moreover, an example is given to show that

$$
\omega_{1}(T(x), T(y)) \leq \omega_{1}(x, y)
$$

but $T$ is not Lipschitzian with respect to $d_{\omega}$ with constant 1 . 
Definition 3.2 Let $(X, \omega)$ be a modular metric space and $M$ be a nonempty subset of $X_{\omega}$. A multivalued mapping $T: M \rightarrow C B(M)$ is called

(i) an $\omega$-contraction if there exists a constant $k \in[0,1)$ such that for any $x, y \in M$,

$$
H_{\omega}(T(x), T(y)) \leq k \omega_{1}(x, y)
$$

(ii) a $(\varepsilon, k)$ - $\omega$-uniformly locally contraction if there exists a constant $k \in[0,1)$ such that for any $x, y \in M$,

$$
H_{\omega}(T(x), T(y)) \leq k \omega_{1}(x, y), \quad \text { whenever } \omega_{1}(x, y)<\varepsilon .
$$

Before we state our results, we will need the following technical lemmas [7] in the setting of modular metric spaces.

Lemma 3.1 Let $(X, \omega)$ be a modular metric space and $M$ be a nonempty subset of $X_{\omega}$. Let $A, B \in C B(M)$, then for each $\varepsilon>0$ and $x \in A$, there exists $y \in B$ such that

$$
\omega_{1}(x, y) \leq H_{\omega}(A, B)+\varepsilon .
$$

Moreover, if $B$ is $\omega$-compact and $\omega$ satisfies the Fatou property, then for any $x \in A$, there exists $y \in B$ such that

$$
\omega_{1}(x, y) \leq H_{\omega}(A, B)
$$

Proof The proof of the first part is easy. As for the second part, assume $B$ is $\omega$-compact and $\omega$ satisfies the Fatou property. Let $x \in A$. Then for any $n \geq 1$, there exists $y_{n} \in B$ such that

$$
\omega_{1}\left(x, y_{n}\right) \leq H_{\omega}(A, B)+\frac{1}{n} .
$$

Since $B$ is $\omega$-compact, we may assume that $\left\{y_{n}\right\} \omega$-converges to $y \in B$. Since $\omega$ satisfies the Fatou property, we get

$$
\omega_{1}(x, y) \leq \liminf _{n \rightarrow \infty} \omega_{1}\left(x, y_{n}\right) \leq H_{\omega}(A, B) .
$$

Lemma 3.2 Let $(X, \omega)$ be a modular metric space. Assume that $\omega$ satisfies $\Delta_{2}$-condition. Let $M$ be a nonempty subset of $X_{\omega}$. Let $A_{n}$ be a sequence of sets in $C B(M)$, and suppose $\lim _{n \rightarrow \infty} H_{\omega}\left(A_{n}, A_{0}\right)=0$ where $A_{0} \in C B(M)$. Then if $x_{n} \in A_{n}$ and $\lim _{n \rightarrow \infty} x_{n}=x_{0}$, it follows that $x_{0} \in A_{0}$.

Proof Using Lemma 3.1, for every $n \geq 1$, there exists $y_{n} \in A_{0}$ such that

$$
\omega_{1}\left(x_{n}, y_{n}\right) \leq H_{\omega}\left(A_{n}, A_{0}\right)+\frac{1}{n} .
$$

Hence

$$
\omega_{2}\left(y_{n}, x_{0}\right) \leq \omega_{1}\left(x_{n}, y_{n}\right)+\omega_{1}\left(x_{n}, x_{0}\right) \leq \omega_{1}\left(x_{n}, x_{0}\right)+H_{\omega}\left(A_{n}, A_{0}\right)+\frac{1}{n},
$$


which implies $\lim _{n \rightarrow \infty} \omega_{2}\left(y_{n}, x_{0}\right)=0$. Since $\omega$ satisfies the $\Delta_{2}$-condition, we have $\lim _{n \rightarrow \infty} \omega_{1}\left(y_{n}, x_{0}\right)=0$. Since $A_{0}$ is $\omega$-closed, we have $x_{0} \in A_{0}$.

\section{The main results}

The statement of Nadler's fixed point result [7] in modular metric spaces is as follows.

Theorem 4.1 Let $(X, \omega)$ be a modular metric space. Assume that $\omega$ is a convex regular modular which satisfies the $\Delta_{2}$-condition. Let $M$ be a nonempty $\omega$-complete subset of $X_{\omega}$. Let $T: M \rightarrow C B(M)$ be an $\omega$-contraction map. Then $T$ has a fixed point.

Proof Since $T$ is an $\omega$-contraction, there exists a constant $k \in[0,1)$ such that for any $x, y \in$ $M$, we have

$$
H_{\omega}(T(x), T(y)) \leq k \omega_{1}(x, y)
$$

Let $x_{0}$ be an arbitrary but fixed element of $M$ and $x_{1} \in T\left(x_{0}\right)$, then from Lemma 3.1, there exists $x_{2} \in T\left(x_{1}\right)$ such that

$$
\omega_{1}\left(x_{1}, x_{2}\right) \leq H_{\omega}\left(T\left(x_{0}\right), T\left(x_{1}\right)\right)+k^{2} .
$$

Similarly, there exists $x_{3} \in T\left(x_{2}\right)$ such that

$$
\omega_{1}\left(x_{2}, x_{3}\right) \leq H_{\omega}\left(T\left(x_{1}\right), T\left(x_{2}\right)\right)+k^{4} .
$$

By induction we build $\left\{x_{n}\right\}$ such that

$$
\omega_{1}\left(x_{n+1}, x_{n}\right) \leq H_{\omega}\left(T\left(x_{n}\right), T\left(x_{n-1}\right)\right)+k^{2 n},
$$

and $x_{n+1} \in T\left(x_{n}\right)$, for every $n \geq 0$. Since $T$ is a contraction, we get

$$
\omega_{1}\left(x_{n+1}, x_{n}\right) \leq k \omega_{1}\left(x_{n}, x_{n-1}\right)+k^{2 n},
$$

for every $n \geq 0$. Hence

$$
\omega_{1}\left(x_{n+1}, x_{n}\right) \leq k^{n}\left(\omega_{1}\left(x_{1}, x_{0}\right)+\frac{1}{1-k}\right),
$$

for every $n \geq 0$. The technical Lemma 2.2 implies that $\left\{x_{n}\right\}$ is $\omega$-Cauchy. Since $M$ is $\omega$-complete, $\left\{x_{n}\right\} \omega$-converges to some point $x_{0} \in M$. Since

$$
H_{\omega}\left(T\left(x_{n}\right), T\left(x_{0}\right)\right) \leq k \omega_{1}\left(x_{n}, x_{0}\right)
$$

we conclude that $\lim _{n \rightarrow \infty} H_{\omega}\left(T\left(x_{n}\right), T\left(x_{0}\right)\right)=0$. Since $x_{n+1} \in T\left(x_{n}\right)$, Lemma 3.2 implies $x_{0} \in$ $T\left(x_{0}\right)$, i.e. $x_{0}$ is fixed point of $T$. This completes the proof of Theorem 4.1.

Edelstein [8] has extended the classical fixed point theorem for a contraction to the case when $X$ is a complete $\varepsilon$-chainable metric space and the mapping $T: X \rightarrow X$ is an $(\varepsilon, k)$ uniformly locally contraction. This result was extended by Nadler [7] to multivalued mappings. Here we investigate Nadler's result in modular metric spaces. First let us introduce 
the $\varepsilon$-chainable concept in modular metric spaces. Our definition is slightly different from the one used in the classical metric spaces since the modular functions fail in general the triangle inequality.

Definition 4.1 Let $(X, \omega)$ be a modular metric space. A nonempty subset $M \subset X_{\omega}$ is said to be finitely $\varepsilon$-chainable (where $\varepsilon>0$ is fixed) if and only if there exists $N \geq 1$ such that for any $a, b \in M$ there is an $N, \varepsilon$-chain from $a$ to $b$ (that is, a finite set of points $x_{0}, x_{1}, \ldots, x_{N} \in$ $M$ such that $x_{0}=a, x_{N}=b$, and $\omega_{1}\left(x_{i}, x_{i+1}\right)<\varepsilon$, for all $\left.i=0,1,2, \ldots, N-1\right)$.

We have the following result.

Theorem 4.2 Let $(X, \omega)$ be a modular metric space. Assume that $\omega$ is a convex regular modular which satisfies the $\Delta_{2}$-type condition and the Fatou property. Let $M$ be a nonempty $\omega$-complete and $\omega$-bounded subset of $X_{\omega}$, which is finitely $\varepsilon$-chainable, for some fixed $\varepsilon>0$. Let $T: M \rightarrow K(M)$ be an $(\varepsilon, k)-\omega$-uniformly locally contraction map. Then $T$ has a fixed point in $M$.

Proof Since $M$ is finitely $\varepsilon$-chainable, there exists $N \geq 1$ such that for any $a, b \in M$ there is a finite set of points $x_{0}, x_{1}, \ldots, x_{N} \in M$ such that $x_{0}=a, x_{N}=b$, and $\omega_{1}\left(x_{i}, x_{i+1}\right)<\varepsilon$, for all $i=0,1,2, \ldots, N-1$. For any $x, y \in M$ define

$$
\omega^{*}(x, y)=\inf \left\{\sum_{i=0}^{i=N-1} \omega_{1}\left(x_{i}, x_{i+1}\right)\right\},
$$

where the infimum is taken over all $N, \varepsilon$-chains $x_{0}, x_{1}, \ldots, x_{N}$ from $x$ to $y$. Our assumptions imply that $\omega^{*}(x, y)<\infty$, for any $x, y \in M$. Using the basic properties of $\omega$, we get

$$
\omega_{N}(x, y) \leq \omega^{*}(x, y)
$$

for any $x, y \in M$. Moreover, if $\omega_{1}(x, y)<\varepsilon$, then we have $\omega^{*}(x, y) \leq \omega_{1}(x, y)$, for any $x, y \in M$. Fix $x \in M$. Set $z_{0}=x$. Choose $z_{1} \in T\left(z_{0}\right)$. Let $x_{0}, \ldots, x_{N}$ be an $N$, $\varepsilon$-chain from $z_{0}$ to $z_{1}$. Such an $N, \varepsilon$-chain exists since $M$ is finitely $\varepsilon$-chainable. Using Lemma 3.1, there exists $y_{1} \in T\left(x_{1}\right)$ such that $\omega_{1}\left(z_{0}, y_{1}\right) \leq H\left(T\left(z_{0}\right), T\left(x_{1}\right)\right)$. Repeating this process we find $y_{2}, \ldots, y_{N}$ such that $y_{i} \in T\left(x_{i}\right)$ and $\omega_{1}\left(y_{i}, y_{i+1}\right) \leq H\left(T\left(x_{i}\right), T\left(x_{i+1}\right)\right)$. It is easy to check that $z_{0}, y_{1}, \ldots, y_{N}$ is an $N, \varepsilon$-chain from $z_{0}$ to $y_{N} \in T\left(z_{1}\right)$. Set $y_{N}=z_{2}$. Using the fact that $T$ is an $(\varepsilon, k)-\omega$ uniformly locally contraction map, we get

$$
\omega^{*}\left(z_{1}, z_{2}\right) \leq k \omega^{*}\left(z_{0}, z_{1}\right)
$$

By induction, we construct a sequence $\left\{z_{n}\right\} \in M$ such that

$$
\omega^{*}\left(z_{n}, z_{n+1}\right) \leq k \omega^{*}\left(z_{n-1}, z_{n}\right)
$$

and $z_{n+1} \in T\left(z_{n}\right)$, for any $n \geq 1$. Obviously we have $\omega^{*}\left(z_{n}, z_{n+1}\right) \leq k^{n} \omega^{*}\left(z_{0}, z_{1}\right)$, for any $n \geq 1$. Since $\omega$ satisfies the $\Delta_{2}$-type condition, there exists $C>0$ such that

$$
\omega_{1}\left(z_{n}, z_{n+1}\right) \leq C \omega_{N}\left(z_{n}, z_{n+1}\right) \leq C \omega^{*}\left(z_{n}, z_{n+1}\right) \leq C k^{n} \omega^{*}\left(z_{0}, z_{1}\right),
$$


for any $n \geq 1$. Lemma 2.2 implies that $\left\{z_{n}\right\}$ is $\omega$-Cauchy. Since $M$ is $\omega$-complete, $\left\{z_{n}\right\}$ $\omega$-converges to some $z \in M$. We claim that $z$ is a fixed point of $T$. Indeed using the ideas developed above, there exists $y_{n} \in T(z)$ such that

$$
\omega^{*}\left(x_{n+1}, y_{n}\right) \leq k \omega^{*}\left(x_{n}, z\right)
$$

for any $n \geq 1$. Since

$$
\omega_{N+1}\left(y_{n}, z\right) \leq \omega_{1}\left(x_{n+1}, z\right)+\omega_{N}\left(x_{n+1}, y_{n}\right) \leq \omega_{1}\left(x_{n+1}, z\right)+k \omega^{*}\left(x_{n}, z\right)
$$

for any $n \geq 1$, we conclude that $\left\{y_{n}\right\} \omega$-converges to $z$. Since $T(z)$ is $\omega$-closed, we get $z \in$ $T(z)$.

\section{Competing interests}

The authors declare that they have no competing interests.

\section{Authors' contributions}

All authors participated in the design of this work and performed equally. All authors read and approved the final manuscript.

\section{Author details}

'Department of Mathematics, King Abdulaziz University, P.O. Box 80203, Jeddah, 21589, Saudi Arabia. ${ }^{2}$ Department of Mathematical Sciences, University of Texas at El Paso, El Paso, TX, USA. ${ }^{3}$ Department of Mathematics and Statistics, King Fahd University of Petroleum and Minerals, Dhahran, 31261, Saudi Arabia.

\section{Acknowledgements}

The first author was supported by the Deanship of Scientific Research (DSR), King Abdoulaziz University. The author, therefore, acknowledge with thanks DSR.

Received: 1 September 2014 Accepted: 12 November 2014 Published: 22 Dec 2014

\section{References}

1. Chistyakov, W: Modular metric spaces, I: basic concepts. Nonlinear Anal. 72(1), 1-14 (2010)

2. Chistyakov, W: Modular metric spaces, II: application to superposition operators. Nonlinear Anal. 72(1), 15-30 (2010)

3. Nakano, H: Modulared Semi-Ordered Linear Spaces. Maruzen, Tokyo (1950)

4. Musielak, J: Orlicz Spaces and Modular Spaces. Lecture Notes in Math., vol. 1034. Springer, Berlin (1983)

5. Orlicz, W: Collected Papers. Parts I, II. PWN, Warsaw (1988)

6. Abdou, AAN, Khamsi, MA: Fixed point results of pointwise contractions in modular metric spaces. Fixed Point Theory Appl. 2013, 163 (2013)

7. Nadler, SB Jr.: Multi-valued contraction mappings. Pac. J. Math. 30, 475-488 (1969)

8. Edelstein, M: An extension of Banach's contraction principle. Proc. Am. Math. Soc. 12, 7-10 (1961)

9. Mizoguchi, N, Takahashi, W: Fixed point theorems for multivalued mappings on complete metric spaces. J. Math. Anal. Appl. 141(1), 177-188 (1989)

10. Reich, S: Some fixed point problems. Atti Accad. Naz. Lincei, Rend. Cl. Sci. Fis. Mat. Nat. 57, 194-198 (1974)

11. Kutbi, MA, Latif, A: Fixed points of multivalued maps in modular function spaces. Fixed Point Theory Appl. 2009, Article ID 786357 (2009). doi:10.1155/2009/786357

12. Khamsi, MA, Kirk, WA: An Introduction to Metric Spaces and Fixed Point Theory. Wiley, New York (2001)

13. Kozlowski, WM: Modular Function Spaces. Series of Monographs and Textbooks in Pure and Applied Mathematics, vol. 122. Dekker, New York (1988)

14. Dominguez Benavides, T, Khamsi, MA, Samadi, S: Uniformly Lipschitzian mappings in modular function spaces Nonlinear Anal., Theory Methods Appl. 46(2), 267-278 (2001)

15. Khamsi, MA, Kozlowski, WK, Reich, S: Fixed point theory in modular function spaces. Nonlinear Anal. 14, 935-953 (1990) 